\title{
Artificial Intelligence: A New Paradigm for Pharmaceutical Applications in Formulations Development
}

\author{
Nagalakshmi Sethuraman \\ Department of Pharmaceutics, Sri Ramachandra Faculty of Pharmacy, Sri Ramachandra Institute of Higher Education and Research, \\ Porur, Chennai, Tamil Nadu, INDIA.
}

\begin{abstract}
Artificial Intelligence is a branch of engineering that specializes in developing intelligent machines. The utilization of artificial intelligence in pharmaceutical technology has elevated over the years. Over the beyond few years, the sector of artificial intelligence has moved from largely theoretical research to real-international applications. Machine learning is an application of artificial intelligence that gives systems the capacity to automated learn and enhance from experience without being especially programmed. Machine learning (MI) ought to protect medicine around $\$ 100$ billion yearly due to more efficiencies in clinical trials, higher insights for decision-making and innovative tools that could assist consumers, physicians, regulators and insurers to make informed decisions. Neural networks, fuzzy logic and genetic algorithms are unexpectedly developing technologies that would be applied to the formulation and manufacturing of pharmaceutical products. The present article, in brief describes the significance of Al within the manner of drug development after which seems on the numerous Al tools that are available at the disposal of a present-day pharmacist to aid in more efficient functioning.
\end{abstract}

Key words: Artificial intelligence, Pharmaceutical technology, Drug development, Health care, Engineering.

\section{INTRODUCTION}

Artificial Intelligence is defined as branch of computer science this is entirely involved with problem-solving ${ }^{1}$ and constructing machines able to deal with tasks that might in other case demand intelligence and human operators. Numerous famous technologies fall with in side the rubric of $\mathrm{AI}$, which incorporates machine learning, deep learning, chatbots, non-linear grid systems and self-modifying graph systems. Artificial Intelligence (AI) has streamlined and impacted the pharmaceutical enterprise in numerous ways, starting from generating new and better drugs to combatting fast-growing diseases. ${ }^{2}$ Artificial intelligence (AI) in Pharma refers to the usage of automatic algorithms to carry out tasks that historically rely upon human intelligence. Over the last 5 years, the utilization of artificial intelligence in the biotech and pharma industry has redefined how scientists formulate new medicines, tackle disease and more. The algorithms, which are a group of rules to be pursued towards calculating or perform problem-solving operations the use of computing devices, are essential to the designing of the AI architecture for process industry. Examples for unexpectedly developing technology are Neural networks, DeepChem, AutoQSAR packages from Schrodinger software and Deep Signal for genomics. The Neural Network creates and evaluates a number of models to discover the one that will best fit the experimental data presented to it. ${ }^{3}$ The use of technology can save money and time at the same time as supplying higher expertise in the relationships among different formulation and process parameters. ${ }^{4}$
Submission Date: 05-05-2020; Revision Date: 14-07-2020; Accepted Date: 16-09-2020

DOI: 10.5530/ijper.54.4.176 Correspondence:

Dr. S. Nagalakshmi Department of

Pharmaceutics, Sri Ramachandra Faculty of Pharmacy, Sri Ramachandra Institute of Higher Education and Research, Porur, Chennai-600116, Tamil Nadu, INDIA.

Phone no: +919176468060 Email id:

nagalakshmimpharm@gmail. com

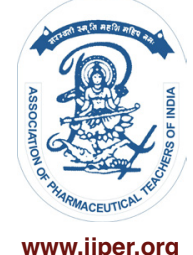




\section{APPLICATION OF AI IN PHARMACEUTICAL RESEARCH}

\section{A.I in the Formulation of Controlled-release tablets}

Artificial neural network (ANN) and pharmacokinetic simulations are utilized for the design of controlledrelease formulations. ${ }^{5} 7$ formulation variables and 3 alternative tablet variables like hardness, particle size and moisture for 22 tablet formulations of a model drug have been used because of the ANN model inputs. In vitro cumulative percentage of drug released at 10 distinct sampling evaluation time has been used as outputs. The ANN model becomes advanced and skilled from the input and the output data units the use of Chem software. The skilled ANN model becomes used to foresee the most effective tablet compositions primarily based on 2 desirable in vitro dissolution-time profiles and 2 desired in vivo release profiles. The dissolution is the rate-limiting step within the in vivo absorption of the drug that the portion of the drug absorbed in vivo is linearly associated with the in vitro dissolution of the drug. In vitro release profiles are observed primarily based on distinction factor, $\mathrm{f} 1, \mathrm{f} 2$ and similarity factor. ${ }^{6}$

\section{A.I in Formulation of Immediate-release tablets}

Turkoglu modelled a direct compression tablet formulation comprising of hydrochlorothiazide so as to improve tablet strength. ${ }^{7}$ In some other study, Kesavan and Peck modelled a tablet formulation of caffeine so as to narrate each formulation (diluting agent and concentration, binder concentration) and processing variables (kind of granulator, the approach of the addition of binder) with granule and tablet characteristics (disintegration time, hardness and friability). Both these examinations confirmed that neural networks performed greater than traditional statistical methods. Consequently, the data of Kesavan and Peck have been re-examined through a sequence of genetic algorithms and neural networks. ${ }^{8}$ This confirmed that the ideal formulation based on the relative significance located at the output characteristics and on the limitations carried out each to the tiers of the elements and to the processing variables.' The identical data had been studied the usage of neuro fuzzy computing. Beneficial regulations have been routinely created, highlighting the utmost essential elements for every asset. ${ }^{5}$

\section{A.I in Product Development}

The Pharmaceutical product development process is a multivariate optimization problem and it involves the optimization of formulation and process variables.
These characteristics make them suitable for solving problems in the area of optimization of formulations in pharmaceutical product development. These models (ANN) provided better fitting and predicting abilities in the development of solid dosage forms in investigations of the effects of several factors such as formulation, compression parameters on tablet physico-chemical properties. It provided a useful tool for the development of micro emulsion-based drug-delivery systems in which experimental effort was minimized. Also, it was used to predict the phase behaviour of quaternary micro emulsion-forming systems consisting of oil, water and two surfactants. ANN finds application to simulate aerosol behaviour, with a view to employing this type of methodology in the evaluation and design of pulmonary drug-delivery systems. Fuzzy logic is a very powerful problem-solving technique for controlling and decision making. The combination of neural networks and fuzzy logic is a potential tool which provides more flexibility and capability to the technique and yields a powerful result. ${ }^{10}$

\section{A.I in Hard Gelatin capsule shell formation development}

Decision-making tool like artificial neural networks (ANN) and Expert Systems (ES) is used for the development of formulations of hard gelatin capsules. ANNs are computer applications that try to arouse certain factors of human thinking namely generalizing, learning, predicting, or abstracting from expertise. With ANNs, the records and statistics initiated at the time of investigational work can be converted highly effortlessly into knowledge, permitting the formulator to at the minimum construct some domain-particular guidelines for future instances or to predict the characteristics of a hypothetical formulation. ${ }^{11}$ In 2005, Wendy I. Wilson developed a capsule shell formulation of BCS class II drugs by expanding the Expert Network and for the analysis. Capsugel's expert system, a centralized system for the formulation of powders in hard gelatin capsules was used worldwide besides its limitation that it provides only a suggested formulation. Drugs such as carbamazepine, ketoprofen, naproxen, diazepam, chlorpropamide and ibuprofen were used as model drugs and these drugs were formulated and tested for their dissolution performance. It was found that at the initial test the system resulted in a high error and poor prediction capabilities. A new data set was determined, the ANN was retrained and the models resulted in $R^{2}$ $\geq 70 \%$. Finally, the intelligent hybrid system predicted the amount of drug dissolved within $5 \%$ for the model drugs. $10 \%$ of the newly developed data were used for cross-validation and it was observed that the developed 
system was able to design a formulation that met its specific performance. Parameters such as wettability and intrinsic dissolution characteristics were addressed by the system and it was shown that the system is suitable for analysis of multiple BCS class II drugs. ${ }^{12}$

\section{Ontology-based expert system for production of the generic immediate-release tablet (OXPIRT)}

Ontology represents knowledge for its explicit specification of a shared conceptualization. To separate among declarative and procedural knowledge, we split knowledge into two types: the domain knowledge and the operation knowledge. The domain knowledge is a declarative knowledge which is represented in ontology. The operation knowledge is a procedural knowledge which is described in production rule. ${ }^{13}$ In 2013, Nopphadol Chalortham et al. used the OXPIRT for the production of immediate-release tablets. This tool provides a better path for a pharmacist to produce a generic immediate-release tablet in a laboratory-scale production. The OXPIRT basically works on two main components Pharmaceutical Tablet Production ontology (PTPO) and Tablet Production Rule (TPR). ${ }^{14}$ Pharmaceutical Tablet Production ontology (PTPO) is created by utilizing an ontology editor called Hozo Environment. ${ }^{15}$ PTPO is the knowledge that contains details of the Active Pharmaceutical Ingredient, excipients, range of concentration, product finishing and other manufacturing processes that were collected from pre-existing data. ${ }^{16}$

TPR is operation-based knowledge that was collected from several cases that work on the API preformulation study and the characteristics of an original tablet such as disintegration time, dissolution profile, etc. In this study atorvastatin calcium, hydroxyzine hydrochloride, metformin $\mathrm{HCl}$ and paracetamol were used in the OXPIRT system. The rules were employed using the TPR and the generic immediate-release tablet was formulated. Based on the rules enlisted, the OXPIRT system concludes with a formulation result that can be produced in the laboratory scale. Then the tablet was formulated and compared with the original product with dissolution profiles and pharmaceutical equivalence that is mandatory for the generic drugs. Based on the evaluated results it was concluded that the OXPIRT system is potential for the successful reformulation of the samples and all the drugs tested show pharmaceutical equivalence to their original products in the first recommendation. Even though this system was potential for immediate-release tablets, it is still a question for other formulations. ${ }^{14}$

\section{CONCLUSION}

Artificial Intelligence is generally defined here as a domain that deals with the design and application of algorithms for analysis of, learning from, and interpretation of data. AI integrates many divisions of machine learning, logic and probability theory, pattern recognition, along with biologically motivated approaches, like neural networks, evolutionary computing or fuzzy modelling, jointly described as "computational intelligence." 17 ANNs are an excellent tool in data analysis and modelling it's stated that a human being is a more advanced machine that will ever be created. Each one would have accepted the present line the last decades ago. However, the scenario, today, has changed drastically. Humans are no longer longer considered the foremost sophisticated machines. The human brain, which is believed to be the most complex network of knowledge, is functioning hard to make something that's for more efficient than a person being in doing any given task and it has succeeded to an excellent extent in doing so. ${ }^{18}$ These tools are capable of working at a way faster rate and therefore the chances of error that will occur with the utilization of those tools are negligible. The larger the health-care sector gets the more sophisticated and more technologically advanced infrastructure it will need. AI not only increases efficiency but also minimizes errors that are much more frequent when a person is handling the task. This, in turn, will mean a decreasing wastage, improved quality of the merchandise, and a bigger margin of profit margin for companies. However, if we look at this from the perspective of human employment, then we will be strained to think that replacing humans for machines will mean large-scale unemployment and shortly all the operations that were once a human job are going to be a part of AI's job. As Stephen Hawking states, "this may mean the end of the human race". Therefore, AI should be introduced into health care but AI should be made such that it works in coordination with humans. ${ }^{19}$

\section{ACKNOWLEDGEMENT}

Author is thankful to Sri Ramachandra Faculty of Pharmacy for providing support in doing the review work.

\section{CONFLICT OF INTEREST}

The author declares no conflict of interest. 


\section{ABBREVIATIONS}

ML: Machine Learning; AI: Artificial Intelligence; QSAR: Quantitative Structure Activity Relationship; ANN: Artificial Neural Network; ES: Expert Systems; BCS: Biopharmaceutical Classification System; OXPIRT: Ontology-based expert system for production of the generic immediate-release tablet; PTPO: Pharmaceutical Tablet Production Ontology; TPR: Tablet Production Rule; API: Active Pharmaceutical Ingredient.

\section{REFERENCES}

1. Manish V. Artificial Intelligence: The beginning of a new Era in Pharmacy Profession. Asian Journal of Pharmaceutics. 2018;12(2):72.

2. What Is Al and How Can It Work for the Pharmaceutical Industry?. Stefanini. com. 2020. [cited 3 August 2020]. Available from: https://stefanini.com/en/ trends/news/a-guide-to-ai-in-the-pharmaceutical-industry

3. Sharma R. Al in pharma: A new perspective. Express Pharma. 2019. [cited 3 August 2020]. Available from: https://www.expresspharma.in/managementpharma/ai-in-pharma-a-new-perspective/

4. Duch W, Swaminathan K, Meller J. Artificial Intelligence Approaches for Rational Drug Design and Discovery. Current Pharmaceutical Design. 2007;13(14):1497-508

5. Ibric S, Djuric Z, Parojcic J, Petrovic J. Artificial intelligence in pharmaceutical product formulation: Neural computing. Chemical Industry and Chemical Engineering Quarterly. 2009;15(4):227-36.

6. Chen Y, McCall T, Baichwal A, Meyer M. The application of an artificial neural network and pharmacokinetic simulations in the design of controlled-release dosage forms. Journal of Controlled Release. 1999;59(1):33-41.

7. Prital S, Khanvilkar VV. Pharmaceutical applications of Artificial intelligence. Int J Pharma Res Health Sci. 2018;6(2):2342-45.
8. Kesavan J, Peck G. Pharmaceutical Granulation and Tablet Formulation Using Neural Networks. Pharmaceutical Development and Technology. 1996;1(4):391-404.

9. Colbourn EA, Rowe RC. Modelling and Optimization of a Tablet Formulation using Neural Networks and Genetic Algorithms. Pharm Technol Eur. 1996;8(9):46-55

10. Arvapalli S, Krishnaveni C, Sharma J, Divya K. Artificial intelligence in pharma industry: A review. International Journal of Innovative Pharmaceutical Sciences and Research. 2019;7(10).

11. Guo M, Kalra G, Wilson W, Peng Y, Augsburge L. A Prototype Intelligent Hybrid System for Hard Gelatin Capsule Formulation Development. Pharmaceutical Technology. 2002;44-52.

12. Wilson W, Peng Y, Augsburger L. Generalization of a prototype intelligent hybrid system for hard gelatin capsule formulation development. AAPS Pharm Sci Tech. 2005;6(3):E449-57.

13. Chalortham N, Leesawat P, Ruangrajitpakorn T, Supnithi T. A Framework of Ontology-Based Tablet Production Supporting System for a Drug Reformulation. IEICE Transactions on Information and Systems. 2011;E94-D(3):448-55.

14. Chalortham N, Ruangrajitpakorn T, Supnithi T, Leesawat P. Oxpirt: Ontologybased expert system for production of a generic immediate release tablet. In: Formulation Tools for Pharmaceutical Development. Elsevier Ltd. 2013;203-28.

15. Nopphadol C. Ontology development for pharmaceutical tablet production expert system., $5^{\text {th }}$ International Conference on Electrical Engineering/ Electronics, Computer, Telecommunications and Information Technology. 2008.

16. Noy N, McGuinness D. Ontology Development 101: A Guide to Creating Your First Ontology. 2020. Protege.stanford.edu. [cited 3 August 2020]. Available from: https://protege.stanford.edu/publications/ontology_development/ ontology101.pdf

17. Man K. F, Tang K. S, Kwong S. Genetic algorithms: concepts and designs, chapter 1-10.

18. IBM. IBM Watson Health. Available from: https:// www.ibm.com/watson/ health/oncology-and-genomics/ oncology/

19. Cellan-Jones R. Stephen Hawking Warns Artificial Intelligence could End Mankind. Available from: http:// www.bbc.com/news/technology-30290540.

Cite this article: Nagalakshmi S. Artificial Intelligence: A New Paradigm for Pharmaceutical Applications in Formulations Development. Indian J of Pharmaceutical Education and Research. 2020;54(4):843-6. 